\title{
Bayesian Estimation of Intra-operative Deformation for Image-Guided Surgery Using 3-D Ultrasound
}

\author{
A.P. King, J.M. Blackall, G.P. Penney, P.J. Edwards, D.L.G. Hill, and D.J. Hawkes \\ Division of Radiological Sciences and Medical Engineering \\ The Guy's, King's and St. Thomas' Schools of Medicine and Dentistry \\ Guy's Hospital, London SE1 9RT, UK \\ andrew.king@kcl.ac.uk
}

\begin{abstract}
This paper describes the application of Bayesian theory to the problem of compensating for soft tissue deformation to improve the accuracy of imageguided surgery. A triangular surface mesh segmented from a pre-operative image is used as the input to the algorithm, and intra-operatively acquired ultrasound data compounded into a 3-D volume is used to guide the deformation process. Prior probabilities are defined for the boundary points of the segmented structure based on knowledge of the direction of gravity, the position of the surface of the surgical scene, and knowledge of the tissue properties. The posterior probabilities of the locations of each of the boundary points are then maximised according to Bayes' theorem. A regularisation term is included to constrain deformation to the global structure of the object.

The technique is demonstrated using a deformable phantom designed to have similar properties to human tissue. Results presented demonstrate that the algorithm was able to recover much of the deformation for a number of objects at varying depths from the source of deformation.

This technique offers a convenient means of introducing prior knowledge of the operative situation into the problem of soft tissue deformation and has the potential for greatly improving the utility of image-guided surgery.
\end{abstract}

\section{Introduction}

Image-guided surgery systems register pre-operative images such as magnetic resonance (MR) or computed tomography (CT) to the physical space of the patient in the operating theatre. Typically surgeons place a tracked pointer into the surgical scene and view the position of the tip of this pointer as three orthogonal slices through the preoperative image on a computer monitor. Recently we described a system which uses an augmented reality (AR) approach to image-guidance [1]. By integrating the surgical microscope into the image-guidance process it is possible to produce accurately aligned overlays in the eye-pieces of the microscope showing critical structures such as arteries or nerves. The surgeon is then able to visualise information from the pre-operative data without having to look away from the surgical scene.

A common problem in any image-guidance system is that of tissue deformation [2] [3]. If tissue has moved, deformed or been resected intra-operatively, the pre-operative image will no longer be an accurate representation of the real surgical scene. Therefore, overlaying information from the image will contain errors. The aim of this paper 
is to present a novel technique for updating the shape and location of a structure segmented from a pre-operative image based on ultrasound data acquired intra-operatively. This will allow the accurate overlaying of information from pre-operative images in the presence of deformation. Previous work related to visualising or compensating for deformation using ultrasound data includes [4] and [5].

Bayesian approaches have been applied in computer vision as a means of introducing prior knowledge to object segmentation and recognition problems which are ill-posed due to high levels of noise (e.g. [6]). A parameterisation of a known object is defined, together with prior probability distributions for the parameter values which indicate the amount of deformation allowed away from the prototypical shape. Bayes' theorem is used to maximise the posterior probability of the object position and shape given noisy image data.

In medical imaging Bayesian techniques have been previously applied in, for example, segmentation of MR images [7]. However, the high levels of noise in ultrasound images would seem to make them ideal data for the application of such techniques. Previous work with this concept includes Kao et al. [8], who used a Bayesian approach for edge detection in single B-mode ultrasound images, including a derivation of a discrete ultrasound imaging model. Lin et al. [9] applied a Bayesian technique to the problem of boundary estimation in compounded 3-D ultrasound volumes, with the aim of producing realistic renderings for visualisation.

This paper describes the application of a Bayesian approach to the problem of compensating for intra-operative tissue deformation using information from a 3-D ultrasound volume. The problem is regularised by the use of a boundary rigidity term. The technique is demonstrated using a deformable phantom designed to have similar elastic properties to human tissue.

\section{Method}

The problem can be stated as follows: given an initial segmentation of an object, in the form of a triangular surface mesh, and intra-operative 3-D ultrasound data of the object, what is the location and shape of the object which maximises the sum of the posterior probabilities of the boundary points subject to the regularisation constraint? In the following text, $\boldsymbol{v}_{i} \in \Re^{3}, i=1 \ldots N$ denotes the initial locations of the boundary points, and the updated positions are denoted by $\boldsymbol{v}_{i}^{\prime} \in \Re^{3}, i=1 \ldots N$.

\subsection{3-D Ultrasound}

True 3-D ultrasound systems directly acquire a 3-D volume using a rectangular array [10]. However, it is thought that the cost and size of the probes currently make their use impractical for intra-operative use. Another approach is to compound a 3-D volume from a set of 2-D ultrasound images. This can be achieved using a number of different methods [10]. All of these approaches require calibration of the ultrasound probe and volume compounding. Several approaches exist for performing the calibration and compounding, and good reviews are provided in [11] and [12] respectively. 
For the work described in this paper a freehand 3-D ultrasound system was used. A Siemens Sonoline Versa Pro ultrasound machine with a $10 \mathrm{MHz}$ probe was calibrated using a cross-wire phantom. The residual error from the calibration process was $0.67 \mathrm{~mm}$. The probe was tracked during acquisition using an Optotrak 3020 optical tracking system from Northern Digital Inc, which tracks infra-red LEDs to a 3-D spatial accuracy of $\sim 0.2 \mathrm{~mm}$. The 2-D slices were compounded into a 3-D volume using the pixel nearest neighbour technique. This algorithm takes every pixel intensity in each 2-D ultrasound image, and places it into the nearest voxel in the final volume image. If more than one intensity is placed into the same voxel, the conflict is resolved by averaging the values concerned.

\subsection{Bayesian Estimation}

The problem of updating the position of each boundary point in a segmented structure to compensate for intra-operative deformation can be expressed in Bayesian terms. Given prior probabilities $P\left(\boldsymbol{v}_{i}^{\prime}=\boldsymbol{x}\right)$ for each boundary point $\boldsymbol{v}_{i}^{\prime}$, and the ultrasound data $I(\boldsymbol{x})$, the problem becomes one of maximising the posterior probability of a boundary being present given the data. Using Bayes' theorem, this posterior probability for a boundary element $i$ is given by

$$
P\left(\left(\boldsymbol{v}_{i}^{\prime}=\boldsymbol{x}\right) \mid I(\boldsymbol{x})\right)=\frac{P\left(I(\boldsymbol{x}) \mid \boldsymbol{v}_{i}^{\prime}=\boldsymbol{x}\right) P\left(\boldsymbol{v}_{i}^{\prime}=\boldsymbol{x}\right)}{P(I(\boldsymbol{x}))}
$$

The prior probability of the image data $P(I(\boldsymbol{x}))$ is assumed to be uniform. The probability of the data given that there is a boundary present, $P\left(I(\boldsymbol{x}) \mid \boldsymbol{v}_{i}^{\prime}=\boldsymbol{x}\right)$, is the imaging model, which can be expressed as a function of the 3-D ultrasound image $f_{U S}(\boldsymbol{x})$. Depending on the type of tissue involved, either the image intensity, the gradient of the image intensity, or a weighted combination of the two may be more appropriate for this model. For example, gradient information should be used at boundaries between tissue types which have different degrees of scatter but similar acoustic impedance, whereas at the boundaries between tissue types with similar scatter but differing acoustic impedance, the ultrasound image intensity will be more appropriate. Prior knowledge is introduced here in the form of a weighting factor $k_{m}$ which determines the proportions of intensity and gradient information used.

$$
P\left(I(\boldsymbol{x}) \mid \boldsymbol{v}_{i}^{\prime}=\boldsymbol{x}\right)=k_{m} f_{U S}(\boldsymbol{x})+\left(1-k_{m}\right)\left\|\nabla f_{U S}(\boldsymbol{x})\right\|
$$

In many applications, the maximum value for the prior probability $P\left(\boldsymbol{v}_{i}^{\prime}=\boldsymbol{x}\right)$ might be taken to be at the initial boundary location, with the probability falling away by some function of distance from this initial position. However, this is a term in which significant prior knowledge of the operative situation can be utilised. As well as the initial location of the boundary point, it is not unreasonable to suppose that knowledge of the direction of gravity and the position and orientation of the surface of the operative scene will also be available. These values can be used to bias the prior probability field. For example, deep structures are likely to shift less than superficial ones after a craniotomy [13], and deformation is most likely to occur in the direction of gravity. 
Therefore, the prior probability field for a given boundary point is given by

$$
P\left(\boldsymbol{v}_{i}^{\prime}=\boldsymbol{x}\right)=F_{\text {prior }}\left(\boldsymbol{g}, \boldsymbol{v}_{i}^{\prime}, \boldsymbol{v}_{i}, \boldsymbol{s}_{p}, \boldsymbol{s}_{n}, d_{e}\right)
$$

where $\boldsymbol{g}$ is a vector indicating the direction of gravity. The surface of the surgical scene is modelled as a plane defined by the point $s_{p}$ and the normal $s_{n}$. The amount of deformation observed at the surface in the direction of gravity is denoted by $d_{e}$.

Hence the maximum prior probability will occur beneath the initial position of the point in the direction of gravity, at a displacement dependent on its distance from the surface and the amount of deformation observed at the surface.

\subsection{Regularisation}

If the above approach were used in isolation there would be no constraint to preserve the global structure of the object and each boundary point would be free to move to its most probable location individually. To constrain the solution so that excessive deformation does not occur, a regularisation term is included and the problem expressed as one of energy minimisation. The total energy of the system is given by

$$
E=(1-\lambda) E_{i}+\lambda E_{r}
$$

where $\left.E_{i}=-P\left(\boldsymbol{v}_{i}^{\prime}=\boldsymbol{x}\right) \mid I(\boldsymbol{x})\right)$ as given in (1), and $\lambda$ is the regularisation weighting.

The rigidity term $E_{r}$ of the energy equation represents the degree to which the global shape of the structure has deformed. The degree of deformation which is permitted will vary depending on the tissue type of the structure. For example, soft tissue such as white matter in the brain should be allowed to deform more than some tumours which will have a greater degree of rigidity. Therefore, prior knowledge of the tissue type can be utilised here in the form of the weighting factor $\lambda$ in (4). The rigidity energy is given by

$$
E_{r}=\sqrt{\sum_{i=1}^{N}\left\|\left(\boldsymbol{v}_{i}^{\prime}-\boldsymbol{v}_{i}\right)-\frac{1}{N} \sum_{i=1}^{N}\left(\boldsymbol{v}_{i}^{\prime}-\boldsymbol{v}_{i}\right)\right\|^{2}}
$$

This expression is the standard deviation of each boundary point's movement away from the mean movement over the whole structure. Hence a shift of the entire structure is not penalised, whereas structural deformation is. This expression allows a certain amount of deformation away from the initial shape, but penalises excessive deformation.

\subsection{Search Strategy}

The global energy minimum represents the state of the object boundary which maximises the sum of the posterior probabilities of its boundary points, subject to the regularising rigidity constraint. This minimum is found using a gradient descent search strategy: the change in posterior probability resulting from each point $\boldsymbol{v}_{i}^{\prime}$ moving in 
each of 6 directions is calculated. The movement that causes the maximum increase in the posterior probability for each point is chosen. Each point is allowed to move if it decreases the energy $E$ after the regularisation constraint is considered.

Since segmented surface meshes typically contain a large number of boundary points, each of which must be allowed to seek its maximum posterior probability, the search for the optimal position and shape takes place in a high-dimensional space which may have many local minima. To reduce the search time, a two-phase technique is employed: each boundary point is first allowed to maximise its prior probability without reference to the data, then each point maximises its posterior probability according to (1). Both phases use the rigidity constraint described in Section 2.3. In addition to this, to enable a larger capture range without a significant increase in computational complexity, a multiresolution approach is used. The ultrasound image volume is smoothed with a $5 \times 5 \times 5$ Gaussian filter and subsampled to produce a series of successively coarser representations of the image volume. The search begins with a relatively large step size at a coarse scale. The step size and coarseness of the ultrasound data is gradually decreased to enable the boundary estimate to be refined through scale space. The search stops when the boundary points have reached a stable energy state.

\section{A Deformable Phantom}

To demonstrate the technique a deformable phantom was constructed (see Figure 1). The phantom material used was gelatine ( $15 \%$ gelatine $-85 \%$ water by weight) as this has been demonstrated to have similar elastic properties to human tissue [14]. Regions of internal structure showing different MR and ultrasound contrast to the structural gelatine were made by adding silica to the gelatine mixture. These regions were immersed into the structural gelatine which was set and placed in a perspex box, with a moveable perspex plate at one end. By turning a number of perspex screws the plate moves and deforms the gelatine block. MR images of the phantom were acquired before deformation (for segmenting the initial structures) and afterwards (to measure the deformation). The phantom was scanned freehand with a tracked calibrated ultrasound probe after deformation.

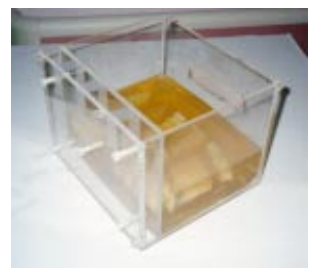

(a)

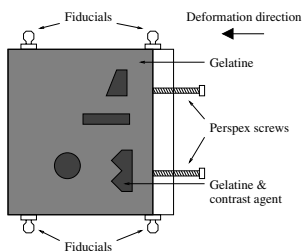

(b)

Fig. 1. (a) The deformable gelatine phantom, (b) diagram showing plan view of phantom

A number of fiducial markers were attached to the phantom prior to MR imaging. These contained a gadolinium solution which gives high image contrast in MR. These 
imaging markers were replaced with physical localisation caps which were localised with a tracked pointer before ultrasound scanning to obtain an MR-physical registration. This registration, together with the tracking and calibration data for the ultrasound probe enabled the ultrasound volume to be aligned with the MR images.

In (3) a general term for the prior probabilities for boundary points was given. The precise form of these priors is dependent on the tissue type. Observations of the nature of deformations present in $15 \%$ concentration gelatine suggested that the amount of deformation has an approximately linear relationship with distance from the source of deformation. Hence the prior probability for boundary point $i$ being in location $\boldsymbol{x}$ is defined by

$$
P\left(\boldsymbol{v}_{i}^{\prime}=\boldsymbol{x}\right)=e^{-\left(\left\|\left(\boldsymbol{v}_{i}^{\prime}-\boldsymbol{v}_{i}\right)-\boldsymbol{g}\left(d_{e}-d_{s} / k_{d}\right)\right\|\right)^{2}}
$$

where $d_{s}=\left(\boldsymbol{v}_{i}^{\prime}-\boldsymbol{s}_{p}\right) \cdot \boldsymbol{s}_{n}$ is the distance of $\boldsymbol{v}_{i}^{\prime}$ beneath the surface of the surgical scene, and $k_{d}$ is a constant which was chosen so that the expected deformation dropped to zero at the wall of the phantom furthest from the perspex plate. The distribution of the prior probabilities is Gaussian, with its peak displaced by the linear term. Work is in progress to model brain deformation more accurately [2][15] and it will be possible to incorporate more realistic models as they become available. In the meantime the simple linear model is compatible with linear compression of an elastic material.

For the purposes of this demonstration, the gravity direction and surface position and normal were all defined manually from the MR images. The direction of gravity was defined as the direction of movement of the perspex plate.

\section{Results}

Figure 2(a) shows a sample slice through the pre-deformed MR image of the phantom overlaid with contours extracted from the deformed MR image. The MR images have an in-plane resolution of $1 \mathrm{~mm} \times 1 \mathrm{~mm}$, and a slice thickness of $1.5 \mathrm{~mm}$. The deformation due to movement of the perspex plate (which was at the bottom of the slice) is apparent. Three structures were segmented from the undeformed MR image using the Analyze software package (Biomedical Imaging Resource, Mayo Foundation, Rochester, MN, USA) and triangle meshes constructed using the marching cubes algorithm. Renderings of these are shown in Figure 2(b). These three structures were chosen because one is close to the deforming plate, and so the deformation would be expected to be large, one was far from the plate, and one object was elongated and covered a range of depth from the plate. Figures 2(c) and (d) show sample slices through the ultrasound volume, and its gradient magnitude image respectively. These images have a voxel size of $1 \mathrm{~mm} \times$ $1 \mathrm{~mm} \times 1 \mathrm{~mm}$.

Figures 3, 4 and 5 show overlays (in dark outlines) onto the post-deformed MR (i.e. the gold standard) of the pre- and post-deformed structures. Sample slices are shown but the deformation is computed in 3-D. It can be seen that in all cases the algorithm has recovered much of the deformation that has occurred. Note that whereas in Figures 3 and 4 the movement of the shape could be approximated by a rigid body motion, in Figure 5 the structure of the shape has deformed significantly owing to the fact that it 


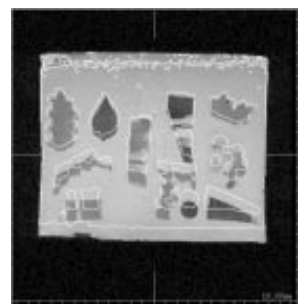

(a)

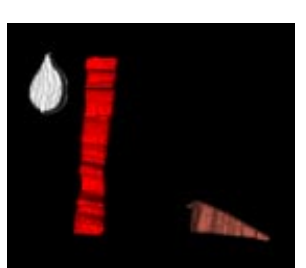

(b)

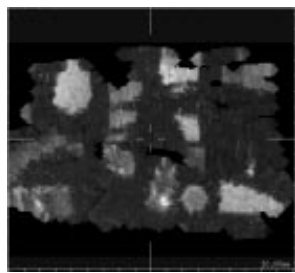

(c)

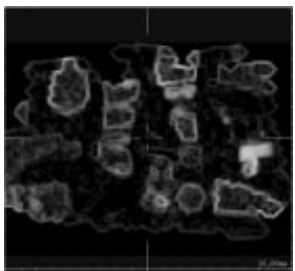

(d)

Fig. 2. The deformable phantom, (a) sample slice through pre-deformation MR image overlaid with contours extracted from post-deformation MR image, (b) renderings of 3 segmented structures from pre-deformation MR image, (c) sample slice through the compounded ultrasound volume, (d) sample slice through the gradient magnitude of the ultrasound volume

extends over a range of depth from the plate. Even in this case the errors in boundary alignment appear to be only $\sim 1-2 \mathrm{~mm}$. However, residual errors do remain. These could arise from errors incurred while calibrating the ultrasound probe, tracking the probe, in the MR-physical registration, or from the assumptions used in calculating the priors.

\section{Discussion}

This paper has outlined a framework for the combination of information from an intraoperative modality with clinical knowledge of the expected surgical deformation to automatically compensate for soft tissue deformation. The technique has its theoretical grounding in Bayesian theory. The results presented here have demonstrated that the technique works well on phantom data. The Bayesian framework is a convenient means of introducing prior knowledge into the problem of deformation compensation, so with more clinically based priors it is not unreasonable to expect good results from clinical data too. This is an area for future investigation. These priors could be taken from measurements of intra-operative deformation, e.g. by using an intra-operative MR scanner [13]. Alternatively, this technique could be combined with a predictive model to provide more realistic priors. For example, finite element models (FEMs) offer a means of constructing a physical model of tissue, which can be used to predict deformation fields given information about the surgical situation [16], such as gravity direction and resection of tissue.

It should be emphasised that the technique described here only compensates for deformation of structures segmented from a pre-operative image modality. No interpolation between these structures is performed. For applications such as pre-operative visualisation or intra-operative augmented reality overlays, this is acceptable. Indeed, it can be seen as an advantage in that relatively sparsely sampled intra-operative image data can be used effectively. However, a more general deformation algorithm would need to provide a deformation field that covers the whole of the surgical scene. Although the current technique does not provide this, the deformation field it provides 


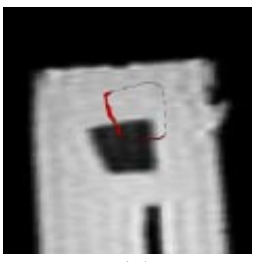

(a)

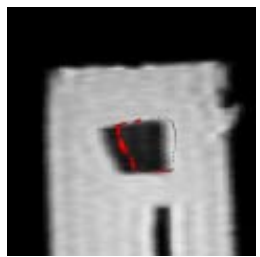

(d)

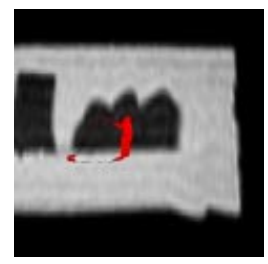

(b)

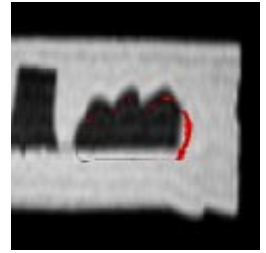

(e)

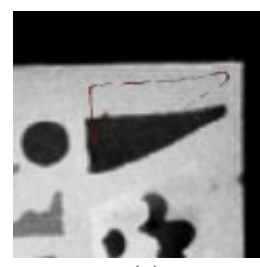

(c)

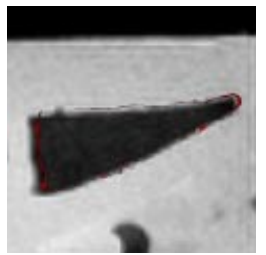

(f)

Fig. 3. Results for shape 1 (a structure near the source of deformation), (a), (b), (c) outlines of undeformed shape on orthogonal slices through the post-deformation MR image, (d), (e), (f) outlines of deformed shape on the post-deformation MR image

could be used as input to an algorithm which does provide such information, such as an FEM [16].

Intra-operative deformation is often caused by resection of tissue, e.g. a tumour. Using the technique described in this paper, information regarding the extent of this resection could be incorporated into the prior probabilities. For example, the probabilities for nearby points could be set relatively high in the area of resection, allowing them to move into it without penalty.

Another important issue in deformation is volume change. Some types of tissue are incompressible, i.e. their volume remains constant under deformation. Other tissue types may undergo volume change under certain conditions. For example, cerebrospinal fluid may drain away, or aedematous regions may increase in volume. Knowledge of the likely compressibility of a structure could be incorporated into the model by including it in the regularisation term.

Another area for future research is the imaging model. The current model defined in (2) does not include knowledge of the ultrasound probe orientation at the time of acquisition. By using this knowledge a more realistic ultrasound imaging model could be incorporated into the framework described here.

The current implementation runs in approximately 5 minutes on a SUN Ultra 10 for an object containing $\sim 1600$ boundary points. Compounding the 3-D ultrasound volume and calculating its gradients is performed separately and takes $\sim 60$ minutes, but there are many ways in which this process could be speeded up. This technique therefore offers the potential for fast intra-operative updates, which give it a significant advantage over many predictive models such as FEMs.

In conclusion, the technique described here is a novel application for Bayesian theory, and has the potential to be able to deal with many of the important issues in defor- 


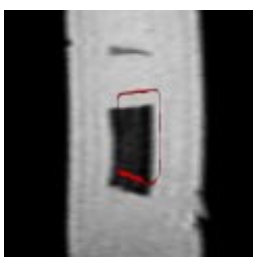

(a)

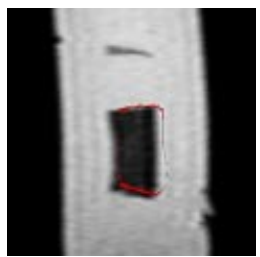

(d)

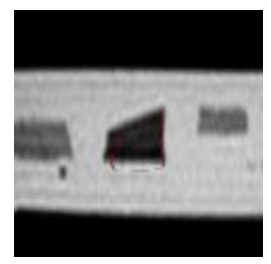

(b)

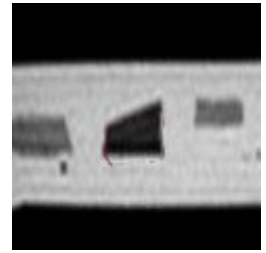

(e)

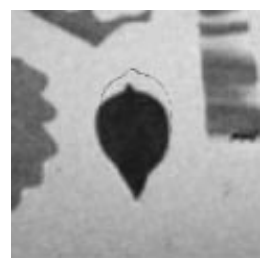

(c)

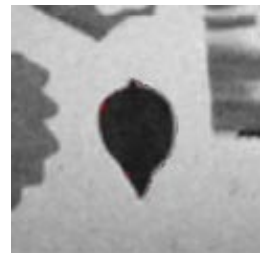

(f)

Fig. 4. Results for shape 2 (a structure far from the source of deformation), (a), (b), (c) outlines of undeformed shape on orthogonal slices through the post-deformation MR image, (d), (e), (f) outlines of deformed shape on the post-deformation MR image

mation. It offers the possibility of improving the utility of image-guidance systems. As such, it represents a highly promising area for future investigation.

\section{Acknowledgements}

We would like to thank the EPSRC for funding this project. We are also grateful to the radiology and radiography staff, and the staff of the medical physics workshop at Guy's hospital for their cooperation.

\section{References}

[1] P. J. Edwards, A. P. King, C. R. Maurer, Jr, D. A. de Cunha, D. J. Hawkes, D. L. G. Hill, R. P. Gaston, M. R. Fenlon, S. Chandra, A. J. Strong, C. L. Chandler, A. Richards, and M. J. Gleeson. Design and evaluation of a system for microscope-assisted guided interventions (MAGI). In Proceedings MICCAI, pages 842-851, 1999.

[2] D. L. G. Hill, C. R. Maurer, R. J. Maciunas, J. M. Barwise, J. A. Fitzpatrick, and M. Y. Wang. Measurement of intraoperative brain surface deformation under a craniotomy. Neurosurgery, 43(3):514-528, 1998.

[3] D. W. Roberts, A. Hartov, F. E. Kennedy, M. I. Miga, and K. D. Paulsen. Intraoperative brain shift and deformation: A quantitative analysis of cortical displacement in 28 cases. Neurosurgery, 43(4):749-760, 1998.

[4] R. D. Bucholz, D. D. Yeh, J. Trobaugh, L. L. McDurmont, C. D. Sturm, C. Baumann, J. M. Henderson, A. Levy, and P. Kessman. The correction of stereotactic inaccuracy caused by brain shift using an intraoperative ultrasound device. In Computer Vision, Virtual Reality, and Robotics in Medicine, pages 459-466, 1997. 


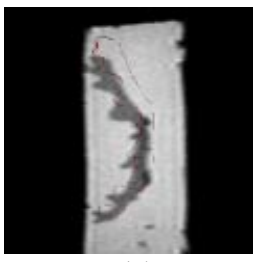

(a)

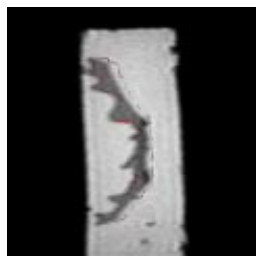

(d)

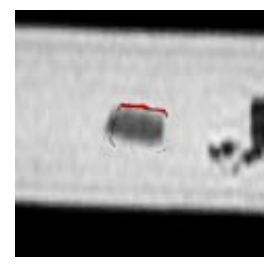

(b)

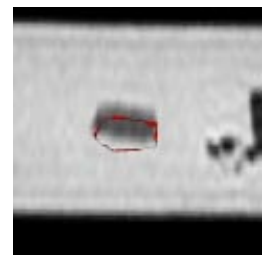

(e)

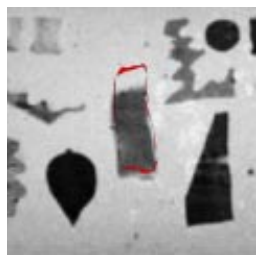

(c)

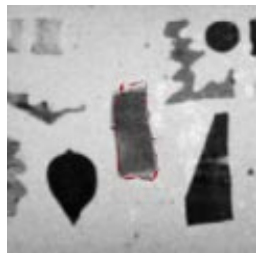

(f)

Fig. 5. Results for shape 3 (a structure covering a range of depth), (a), (b), (c) outlines of undeformed shape on orthogonal slices through the post-deformation MR image, (d), (e), (f) outlines of deformed shape on the post-deformation MR image

[5] D. G. Gobbi, R. M. Comeau, and T. M. Peters. Ultrasound probe tracking for real-time ultrasound/MRI overlay and visualization of brain shift. In Proceedings MICCAI, pages 920-927, 1999.

[6] A. L. Yuille, P. W. Hallinan, and D. S. Cohen. Feature extraction from faces using deformable templates. International Journal of Computer Vision, 8:99-111, 1992.

[7] T. Kapur, E. L. Grimson, R. Kikinis, and W. M. Wells. Enhanced spatial priors for segmentation of magnetic resonance imagery. In Proceedings MICCAI, pages 457-468, 1998.

[8] C-M. Kao, X. Pan, E. Hiller, and C-T. Chen. A Bayesian approach for edge detection in medical ultrasound images. IEEE Transactions on Nuclear Science, 45:3089-3096, 1998.

[9] W. J. Lin, S. M. Pizer, and V. E. Johnson. Boundary estimation in ultrasound images. In Information Processing in Medical Imaging, pages 285-299, 1991.

[10] A. Fenster and D. B. Downey. 3-D ultrasound imaging: A review. IEEE Engineering in Medicine and Biology Magazine, 15(6):41-51, 1996.

[11] R. W. Prager, R. N. Rohling, A. H. Gee, and L. Berman. Rapid calibration for 3-D freehand ultrasound. Ultrasound in Medicine and Biology, 24(6):855-869, 1998.

[12] R. N. Rohling, A. H. Gee, and L. Berman. Radial basis function interpolation for 3-D ultrasound. Technical report, Cambridge University Engineering Department, 1998.

[13] C. R. Maurer, Jr., D. L. G. Hill, A. J. Martin, H. Liu, M. McCue, D. Rueckert, D. Lloret, W. A. Hall, R. E. Maxwell, D. J. Hawkes, and C. L. Truwit. Investigation of intraoperative brain deformation using a 1.5 Tesla interventional MR system: Preliminary results. IEEE Transactions on Medical Imaging, 17:817-825, 1998.

[14] L. K. Ryan and F. S. Foster. Tissue equivalent vessel phantoms for intravascular ultrasound. Ultrasound in Medicine and Biology, 23(2):261-273, 1997.

[15] T Hartkens, D. L. G. Hill, C. R. Maurer, Jr, A. J. Martin, W. A. Hall, D. J. Hawkes, D. Rueckert, and C. L. Truwit. Quantifying the intraoperative brain deformation using interventional MR imaging. In Proceedings ISMRM, 2000.

[16] K. D. Paulsen, M. I. Miga, F. E. Kennedy, P J. Hoopes, A. Hartov, and D. W. Roberts. A computational model for tracking subsurface tissue deformation during stereotactic neurosurgery. IEEE Transactions on Biomedical Engineering, 46:213-225, 1999. 